\title{
Personalized medicine: a new option for nuclear medicine and molecular imaging in the third millennium
}

\author{
Orazio Schillaci $^{1,2} \cdot$ Nicoletta Urbano $^{3}$
}

Published online: 12 January 2017

(C) Springer-Verlag Berlin Heidelberg 2017

"It's more important to know what sort of person has a disease than to know what sort of disease a person has," stated Hippocrates of Kos (c. 460 - c. 370 BC). This sentence of the father of modern medicine, which is based on observation of clinical signs and rational conclusions, is truly actual and it can be considered the manifesto of personalized (or precision) medicine (PM). According to the National Heart, Lung, and Blood Institute, PM is the use of diagnostic and screening methods to better manage the individual patient's disease or predisposition toward a disease; it will enable risk assessment, diagnosis, prevention, and therapy specifically tailored to the unique characteristics of the individual, thus enhancing the quality of life and public health.

It is of the utmost importance to highlight that USA President Barack Obama on January 20, 2015, in his State of the Union address, announced the PM initiative and called for $\$ 215$ million in fiscal year 2016 to support it. President Obama' idea was that through advances in research, technology, and policies which empower patients, the PM initiative will enable a new era of medicine in which researchers, providers, and patients work together to develop individualized care [1].

This Editorial Commentary refers to the article http://dx.doi.org/10.1007 /s00259-016-3580-5.

Orazio Schillaci

orazio.schillaci@uniroma2.it

1 Department of Biomedicine and Prevention, University Tor Vergata, Viale Mazzini 121, 00195 Rome, Italy

2 IRCCS Neuromed, Pozzilli, Italy

3 Nuclear Medicine, Policlinico Tor Vergata, Rome, Italy
The concept of PM, prevention and treatment strategies taking into account individual variability, might be strictly related to molecular imaging and nuclear medicine, through understanding the molecular basis of disease and defining molecular changes or markers associated with disease progression, response to treatment, and relapse. Moreover, PM is one of the components of the so-called P4 medicine, i.e. predictive, preventative, personalized, and participatory, where predictive medicine is the use of diagnostic tests to predict when a disease will become symptomatic, preventative is to take some action to prevent the precursor state from evolving into the symptomatic stage, and participatory is the idea of patients playing an active role in remaining in the wellness state [2]. This new vision of medicine includes systems approaches to biology and medicine: systems biology is the study of biological systems as collections of networks at multiple levels, from the molecular step, through cells, tissues, and organisms, to the population level; systems medicine is the application of systems biology to human disease [3].

In this scenario, molecular imaging can play an important role, because it enables early detection and/or identification of changes occurring in tissue and, consequently, changes in individual patient management in real time, it serves as a noninvasive diagnostic and monitoring tool, it allows researchers to explore new ways to manage and treat illnesses, and it facilitates drug development [4]. As a matter of fact, if PM is mainly based on molecular characterization using genomic and proteomic techniques, these ones are limited in oncology, because, as tumours are spatially and temporally heterogeneous, they require surgery or biopsies to extract and analyze what are generally small portions of cancer tissue that do not allow a full characterization of the neoplasm. On the contrary, imaging is able to non-invasively produce a view of the entire tumour, and it can be used on an ongoing basis and often repeated during treatment [5]. 
Therefore, because medical imaging is really very important for almost all aspects of PM, the research committee of the European Society of Radiology (ESR) has recently publish a white paper on this topic [6]. In this context, imaging genomics (also called radiogenomics) is a new paradigm based on the assumption that the medical images are the product of mechanisms occurring at molecular and genetic levels, in which large sets of complex descriptors of disease are extracted from imaging and related to molecular biology and gene expression patterns of disease. Then, the extracted imaging features, particularly in oncological studies, are correlated to gene expression. Some examples of the possible applications of this approach are: understanding the biological correlates behind image phenotypes, understanding how a biological process is reflected in imaging, and defining clinical biomarkers or biological surrogates [7].

Therefore, identifying imaging biomarkers of the genotype and phenotype of cancer is of the utmost importance for oncologists because this information can provide useful targets for therapy without the need for tissue sampling. Research in the field of radiogenomics has shown the potential of this approach to obtaining tumor genotype and phenotype, also by means of nuclear medicine images.

In 2004, Lee et al. [8] studied the gene expression profiles of 10 patients with hepatocellular carcinomas (HCCs), and compared it with FDG uptake, resulting in high uptake in 4 cases and low uptake in the remaining 6. HCCs with high FDG uptake were pathologically poorly differentiated (Edmondson-Steiner grade III), whereas those showing low uptake were grade II $(n=2)$ or grade II with a focal area of grade III $(n=3)$. One patient with low FDG uptake had a grade III lesion, but his gene expression profile showed features of both the high and the low uptake group. It is worth noting that the high-grade tumors exhibited upregulation of 11 genes, in particular, ones involved in invasion or cell spreading, including vascular cell adhesion molecule- 1 and vinexin beta, and 9 genes were repressed. These findings suggest that HCCs with high FDG uptake appear to have more aggressive biological properties than those with low uptake, because the radiopharmaceutical accumulation is closely related to the molecular markers involved in tumour progression and metastasis. Therefore, FDG-PET might be an important diagnostic tool that would differentiate aggressiveness of HCC and support treatment decisions.

Strauss et al. [9] studied FDG kinetics and gene expression in bone giant cell tumours, which are usually classified as benign lesions, but they frequently show local recurrence and also lung metastases may occur. Tumor specimens obtained from five patients were assessed with gene chip technology and these data were compared with the quantitative FDG findings. All tumors showed significant radiopharmaceutical uptake, mainly due to an enhanced vascular fraction and increased FDG transport. Quantitative data for about 12,626 genes were obtained per gene chip; the expression values for 137 genes $(1.1 \%)$ exceeded the median expression value for the reference gene, i.e. $\beta_{2}$-microglobulin. The highest expression was observed for the gene for the small, leucine-rich proteoglycan I, that has a significant impact on bone cell differentiation and proliferation. Moreover, correlation analysis revealed an association of FDG data with the expression of several genes, in particular, those related to angiogenesis.

The dependency of FDG kinetics on angiogenesis-related gene expression has been subsequently confirmed in 25 patients with primary colorectal cancer (CRC) [10], in whom tissue specimens were obtained from the tumor and the normal colon during surgery, and gene expression was assessed using gene arrays. The results of this study indicated that FDG kinetics are modulated by the expression of angiogenesisrelated genes; in particular, vascular endothelial growth factor A (VEGF-A) was directly correlated with the FDG transport and angiopoietin-2 with the fractal dimension of the 18F-FDG kinetics.

Gastrointestinal stromal tumors (GISTs) are relatively rare tumors of the gastrointestinal tract, in which mutations in the c-KIT gene are found, providing rationale for use of imatinib for their treatment. The results obtained with dynamic PET were compared to gene expression data obtained in 22 patients with GIST [11]. Gene array data were based on tumor specimen obtained by surgery after an FDG-PET scan. FDG uptake was found to be associated with the expression of several zinc finger proteins, the expression of which was related to sensitivity to imatinib [12]; furthermore, the transport of FDG was associated with VEGF-A. These findings suggest that FDGPET may serve as a screening tool to identify those patients with GISTs who are most likely to respond favorably to imatinib treatment.

A subset of 20 locally invasive breast cancers of patients submitted to FDG-PET was analyzed using the human genome U133A oligonucleotide microarray [13]. FDG uptake was significantly and negatively correlated with estrogen receptor (ER) status: ER-positive tumours had lower SUV values than ER-negative ones. Using unsupervised hierarchic clustering of genomewide expression profiles, tumors were grouped into 2 series of 10 each; a fundamental feature affecting grouping was expression of the ER and ER-related genes. In group 1, 9 out of 10 of the tumors were ER-positive by immunohistochemistry, whereas group 2 contained only ERnegative tumors. Moreover, genes that were differentially expressed between the two groups included those involved in glucose metabolism and transport, consistent with strong hypoxia and glycolysis pathways in the ER-negative cancers.

Nair et al. [14] have recently proposed an innovative approach to associate gene expression with prognostic FDG uptake features using three cohorts (study, external, and validation cohorts). The study group included 25 patients with surgically resected non-small cell lung cancer (NSCLC) analyzed 
for tumor FDG uptake features matched with excised tumor specimens subjected to global gene expression analysis, in whom follow-up was not available. Data for the external cohort was derived from a previous study modelling gene expression and outcome in a group of patients with NSCLC [15]. Eighty-three patients made up the validation cohort: they had resected, limited-stage NSCLC, and had undergone treatment-naive, preoperative PET/CT imaging; for all of them, outcome data were available. Both in the study and validation cohorts, preoperative PET/CT images were retrospectively evaluated and FDG uptake was quantified using 14 metrics of interest related to SUV, which were compared with gene expression for single genes and co-expressed gene clusters (metagenes). Predicted FDG uptake features from the study cohort were examined in the external cohort with NSCLC outcome data and gene expression; then, validation of the predicted FDG uptake features identified as prognostic in the external cohort was conducted in the third cohort with PET imaging and outcome data to determine whether the true FDG uptake features remained significantly associated with overall survival. Four out of eight single genes correlated to FDG uptake were also associated with survival. The most prognostic metagene signature was related to a multivariate FDG uptake feature (SUVmaximum, SUV variance and SUV PCA2, derived from principle component analysis), each highly associated with survival in the external cohorts. Moreover, cellcycle, proliferation, death, and self-recognition pathways were altered in this radiogenomic profile. The results of this paper clearly indicate that combining tumour genomics with FDG uptake might be of value for a better understanding of the use of this radiopharmaceutical as a biomarker.

A radiogenomics strategy for associating image features with clusters of co-expressed genes (metagenes) was applied in 26 patients with NSCLC for whom gene expression and 180 image features from CT and PET/CT were available [16]. Then, the prognostic significance of the predicted image features were evaluated in a public gene expression data set with survival outcomes. PET/CT image features and the PET SUV were predicted in terms of metagenes with an accuracy of 65$86 \%$. These results are potentially important for patient care: prognostically significant patient-specific molecular signatures may be predicted non-invasively from image features, further advancing the role of molecular imaging in personalized medicine.

Several studies have shown that KRAS (v-Ki-ras2 Kirsten rat sarcoma viral oncogene homolog) mutations in CRC can cause the lack of response to anti-epidermal growth factor receptor-based therapy [17]. Therefore, KRAS mutational testing has been incorporated into routine clinical practice, but it is limited by the heterogeneity of KRAS status, either intra-tumoural within a primary cancer or discordant status between a primary CRC and its metastases [18]. Kawada et al. reported that FDG accumulation was significantly higher both in primary CRCs with mutated KRAS than in those with wild-type KRAS [19], and in metastatic ones [18], suggesting that FDG PET/CT scans could be useful in the prediction of KRAS mutational status. These findings have been recently confirmed [20]: FDG PET/CT could supplement genomic analysis to determine KRAS expression in CRC; nevertheless, caution is mandatory in order to insure proper patient selection, because severe local inflammation with increased $\mathrm{C}$ reactive protein levels might affect FDG quantification.

The articles cited above are some of the first experiences of correlating nuclear imaging phenotypes with genomics signatures. This issue is really interesting, and, as a matter of fact, the National Cancer Institute Cancer Imaging Program recently organized two related workshops on this topic [21]. Radiogenomics represents a synergy derived from data integration, and it has the potential to impact therapy strategies by identifying more patient-specific prognostics and assessments of response to drug or radiation therapy. However, the correlation of imaging phenotypes with genomics signatures should require technical implementation, with the use of standardized methods for data analysis, feature extraction, and data integration [22].

In this issue of the European Journal of Nuclear Medicine and Molecular Imaging, Chen et al. have studied the correlation of pre-treatment FDG tumour uptake heterogenicity with gene expression profiling in patients with newly diagnosed oropharyngeal and hypopharyngeal cancer who completed radiotherapy or chemoradiotherapy [23]. Their findings indicate that texture features are able to give prognostic information beyond classical PET parameters, such as maximum SUV, metabolic tumour volume, and total lesion glycolysis, allowing a better stratification of treatment outcome. This observation confirms that one treatment does not work for all patients, because the efficacy is related also to the tumor genotype and phenotypic behavior pattern in each individual, and, therefore, it would be of the utmost importance determining which subject may benefit from a different therapy.

The unique ability of nuclear medicine to characterize biological processes at the cellular and molecular levels is bringing increasing attention to our imaging modalities as medicine evolves toward more personalized forms of treatment, and it will become increasingly relevant as personalized healthcare practice grows in modern medicine. Therefore, the integration of functional and molecular images with -omics information can be a powerful strategy, for improving diagnostic accuracy and patient care. In the near future, advances in understanding the genetic basis of disease, combined with the availability of new radiopharmaceuticals, will provide a major opportunity to optimize treatment of several human diseases on an individual level, also in a cost-effective manner.

The implementation of PM would include the promotion of using non-invasive medical imaging, and, in particular, molecular imaging, to allow new treatments for very defined 
groups of patients having determined features, thereby avoiding aggressive treatment in patients in which one specific therapy would not be useful, and cutting costs without cutting the quality of the health care. Nuclear medicine could reveal the underlying cause for a functional imaging phenotype and provide prognostically important information, because molecular images are more than pictures [24] and more than meets the eye [25].

\section{References}

1. Collins FS, Varmus H. A new initiative on precision medicine. $\mathrm{N}$ Engl J Med. 2015;372:793-5.

2. Sobradillo P, Pozo F, Agustí A. P4 medicine: the future around the corner. Arch Bronconeumol. 2011;47:35-40.

3. Flores M, Glusman G, Brogaard K, Price ND, Hood L. P4 medicine: how systems medicine will transform the healthcare sector and society. Per Med. 2013;10:565-76.

4. Jung KH, Lee KH. Molecular imaging in the era of personalized medicine. J Pathol Transl Med. 2015;49:5-12.

5. Kircher MF, Hricak H, Larson SM. Molecular imaging for personalized cancer care. Mol Oncol. 2012;6:182-95.

6. European Society of Radiology (ESR). Medical imaging in personalised medicine: a white paper of the research committee of the European Society of Radiology (ESR). Insights Imaging. 2015;6:141-55.

7. Kuo MD, Jamshidi N. Behind the numbers: Decoding molecular phenotypes with radiogenomics-guiding principles and technical considerations. Radiology. 2014;270:320-5.

8. Lee JD, Yun M, Lee JM, Choi Y, Kim JS, Kim SJ, et al. Analysis of gene expression profiles of hepatocellular carcinomasd with regard to $18 \mathrm{~F}$-fluorodeoxyglucose uptake pattern on positron emission tomography. Eur J Nucl Med Mol Imaging. 2004;31:1621-30.

9. Strauss LG, Dimitrakopoulou-Strauss A, Koczan D, Bernd L, Haberkorn U, Ewerbeck V, et al. 18F-FDG kinetics and gene expression in giant cell tumors. J Nucl Med. 2004;45:1528-35.

10. Strauss LG, Koczan D, Klippel S, Pan L, Cheng C, Willis S, et al. Impact of angiogenesis-related gene expression on the tracer kinetics of 18F-FDG in colorectal tumors. J Nucl Med. 2008;49:123844.

11. Strauss LG, Dimitrakopoulou-Strauss A, Koczan D, Pan L, Hohenberger P. Correlation of dynamic PET and gene array data in patients with gastrointestinal stromal tumors. Sci World J. 2012;2012:721313.

12. Rink L, Ochs MF, Zhou Y, von Mehren M, Godwin AK. ZNFmediated resistance to imatinib mesylate in gastrointestinal stromal tumor. PLoS One. 2013;8:e54477.
13. Osborne JR, Port E, Gonen M, Doane A, Yeung H, Gerald W, et al. F-18-FDG PET of locally invasive breast cancer and association of estrogen receptor status with standardized uptake value: Microarray and immunohistochemical analysis. J Nucl Med. 2010;51:543-50.

14. Nair VS, Gevaert O, Davidzon G, Napel S, Graves EE, Hoang CD, et al. Prognostic PET F-18-FDG uptake imaging features are associated with major oncogenomic alterations in patients with resected non-small cell lung cancer. Cancer Res. 2012;72:3725-34.

15. Lee ES, Son DS, Kim SH, Lee J, Jo J, Han J, et al. Prediction of recurrence-free survival in postoperative non-small cell lung cancer patients by using an integrated model of clinical information and gene expression. Clin Cancer Res. 2008;14:7397-404.

16. Gevaert $\mathrm{O}, \mathrm{Xu}$ J, Hoang CD, Leung AN, Xu Y, Quon A, et al. Nonsmall cell lung cancer: identifying prognostic imaging biomarkers by leveraging public gene expression microarray data-methods and preliminary results. Radiology. 2012;264:387-96.

17. Karapetis CS, Khambata-Ford S, Jonker DJ, O'Callaghan CJ, Tu D, Tebbutt NC, et al. K-ras mutations and benefit from cetuximab in advanced colorectal cancer. N Engl J Med. 2008;359:1757-65.

18. Kawada K, Toda K, Nakamoto Y, Iwamoto M, Hatano E, Chen F, et al. K, Date H, Uemoto S, Sakai Y. Relationship between 18FFDG PET/CT scans and KRAS mutations in metastatic colorectal cancer. J Nucl Med. 2015;56:1322-7.

19. Kawada K, Nakamoto Y, Kawada M, Hida K, Matsumoto T, Murakami T, et al. Relationship between 18F-fluorodeoxyglucose accumulation and KRAS/BRAF mutations in colorectal cancer. Clin Cancer Res. 2012;18:1696-703.

20. Lee JH, Kang J, Baik SH, Lee KY, Lim BJ, Jeon TJ, et al. Relationship between 18F-Fluorodeoxyglucose uptake and V-KiRas2 Kirsten rat sarcoma viral oncogene homolog mutation in colorectal cancer patients: variability depending on C-reactive protein level. Medicine (Baltimore). 2016;95:e2236. doi:10.1097 /MD.0000000000002236.

21. Colen R, Foster I, Gatenby R, Giger ME, Gillies R, Gutman D, et al. NCI workshop report: clinical and computational requirements for correlating imaging phenotypes with genomics signatures. Transl Oncol. 2014;24(7):556-69.

22. Hatt M, Tixier F, Pierce L, Kinahan PE, Cheze Le Rest C, Visvikis D. Characterization of PET/CT images using texture analysis: the past, the present... any future? Eur J Nucl Med Mol Imaging. 2017;44:151-65.

23. Chen SW, Shen WC, Lin YC, Chen RY, Hsieh TC, Yen KY, et al. Correlation of pretreatment (18)F-FDG PET tumor textural features with gene expression in pharyngeal cancer and implications for radiotherapy-based treatment outcomes. Eur J Nucl Med Mol Imaging. 2016. doi:10.1007/s00259-016-3580-5.

24. Gillies RJ, Kinahan PE, Hricak H. Radiomics: images are more than pictures, they are data. Radiology. 2016;278:563-77.

25. Hatt M, Tixier F, Cheze Le Rest C. Radiomics in PET/CT: more than meets the eye? J Nucl Med. 2016. doi:10.2967 /jnumed.116.184655. 